\title{
Lernen durch Engagement
}

\section{Service Learning im Kontext von Zivilgesellschaft}

Gabriele Bartsch

\begin{abstract}
Junge Menschen in Schule, Studium und Ausbildung können durch gesellschaftliches Engagement ibr fachliches Lernen vertiefen. Das Konzept des "Service-Learnings « kombiniert deshalb kognitives Lernen mit der Übernahme von Verantwortung im Schulumfeld.
\end{abstract}

Deutschland verfügt über eine lange Tradition bürgerschaftlichen Engagements, denken wir an die zahlreichen Vereine, deren Entstehung weit in das 19.Jahrhundert zurückreicht. Frank Adloff hat die Entstehung zivilgesellschaftlicher Formen mit den typischen Ausprägungen des Vereinswesens und Ehrenamts in Deutschland gut zusammengefasst (Adloff 2005, S. 100ff.). Auffallend ist, dass in Deutschland seit den 1990er Jahren ein Diskurs über neue zivilgesellschaftliche Engagementformen Einzug gehalten hat, der zeitlich einhergeht mit zurückgehenden Steuereinnahmen öffentlicher Haushalte.

Interessant an dieser Entwicklung ist, dass zunächst versucht wurde, neben den traditionellen Formen ehrenamtlichen Engagements in etablierten Strukturen wie Vereinen und Wohlfahrtsorganisationen, neue Engagementformen zu entwickeln. Das klassische Ehrenamt galt als überholt, nicht umsonst wird gelegentlich mit abfälligem Unterton von der typisch deutschen »Vereinsmeierei « gesprochen. Demgegenüber erschien das zeitlich befristete, projektorientierte freiwillige Engagement attraktiv. Wer sich freiwillig en-

Gabriele Bartsch, Soziologin und Kulturwissenschaftlerin, ist seit dem Jahre 2000 Geschäftsführerin der Agentur mehrwert in Stuttgart. Davor war sie zehn Jahre in der professionellen Frauenförderung tätig, u. a. in der Evang. Landeskirche Württemberg, in dieser Funktion auch Projektleiterin Personalentwicklung.

Internet http://www.agentur-mehrwert.de gagieren wollte, musste plötzlich nicht mehr Vereinsmitglied werden, sondern konnte sich an Freiwilligenagenturen wenden, die auf lokaler Ebene entstanden und die Organisation und Koordination von Einsatzmöglichkeiten übernahmen.

Gleichwohl von den Verfechtern der neuen Freiwilligenarbeit betont wurde, dass ein Werben für mehr bürgerschaftliches Engagement keineswegs als Ersatz für zurückgehendes staatliches Engagement anzusehen ist, ist die Gleichzeitigkeit nicht von der Hand zu weisen. Widersprüchlich ist dabei allerdings, wie schwer sich die Akteure der neuen zivilgesellschaftlichen Organisationen taten und nach wie vor tun, öffentliche Anerkennung und vor allem öffentliche Mittel zu erhalten.

Machen wir uns heute darüber Gedanken, wie die westliche postindustrielle Gesellschaft zukunftsfähig werden kann, fällt der Blick auf das Stichwort Sozialkapital. Der Begriff Sozialkapital beschreibt die Fähigkeit einer Gesellschaft, ihren sozialen Zusammenhalt zu organisieren und aufrechtzuerhalten. Eine gute $\mathrm{Zu}$ sammenfassung dazu hat Holger Backhaus-Maul erstellt (Backhaus-Maul, 2006). Dabei spielen Vertrauen, Regeln und Normen sowie Netzwerke eine zentrale Rolle, mittels all dessen Menschen in die Lage versetzt werden, Herausforderungen und Probleme gemeinschaftlich zu lösen.

Orte der Sozialkapitalbildung sind zum einen Familie und Bildungsinstitutionen, zum anderen alle zivilgesellschaftlichen Formationen, wie Vereine, Verbände, Gruppierungen, Bürgerbewegungen. Also alle Initiativen, deren Zielsetzung über das Interesse von Privatpersonen und kleinen Teilgruppen hinausgeht und in der politischen Definition dem Dritten Sektor zugerechnet werden.

Bei diesen neuen Formen geht es gewissermaßen um eine neue Interaktion zwischen Staat, Wirtschaft und dem dritten Sektor. Zivilgesellschaft soll gewährlei- sten, dass die Aushandlung verschiedener, in der Regel sich widersprechender Interessen, demokratisch legitimiert, gleichberechtigt und friedlich abläuft. Weltweite kriegerische Auseinandersetzungen und Krisenherde weisen uns immer wieder darauf hin, dass das Konzept der Zivilgesellschaft nicht als Freizeitvergnügen unausgelasteter Mittelschichtangehöriger einzustufen ist, sondern existenzielle Voraussetzung und Notwendigkeit demokratiefähiger Gesellschaften sind.

Die zentrale Frage ist: Was vermittelt Menschen angesichts eines dynamischen Wirtschaftssystems und einer temporär und regional möglichen hohen Arbeitslosenrate Sicherheit und was verweist auf Zukunft? Wie kann gewährleistet werden, dass Menschen unterschiedlicher Herkunft, unterschiedlichen Glaubens, unterschiedlicher politischer Auffassungen friedlich und aufeinander bezogen zusammenleben? Was kann eine Zivilgesellschaft beflügeln?

Die Reformvorschläge reichen von neoliberalistischen Ansätzen, die im Rückzug des (Sozial-) Staates eine Stärkung der Selbstverantwortung und der Selbstorganisation des einzelnen Bürgers wähnen (Walzer), bis hin zu partizipativen gesellschaftspolitischen Konzepten, in dem es um neue Rollenbestimmungen zwischen den drei Akteuren Bürger, Staat und Wirtschaft geht. In dieser zivilgesellschaftlichen Variante schimmert ein stark koordinierender und steuernder Staat durch, bei der Rollenbestimmung der Bürgerinnen und Bürger geht es um deren gestalterischen Part in der öffentlichen Aushandlung von Interessen.

Typische Beispiele hierfür sind Initiativgruppen, die sich für Stadtteilprojekte, Frauenprojekte einsetzen oder für die Rechte benachteiligter Gruppen kämpfen. Es ist nur auf den ersten Blick paradox, wenn in dem zivilgesellschaftlichen Entwurf ein starker Staat gefordert wird. Er ist allerdings weniger in seiner büro- 
kratischen Rolle gefragt, sondern in einer starken Steuerungs- und Koordinierungsfunktion, der Aushandlungsprozesse dadurch mit anstößt und mitgestaltet. Es hat den Anschein, als ob die »Wende nach 1989 mit der Politik der Runden Tische eine solche zivilgesellschaftliche Kultur verstärkt hat.

Im Zeitalter des Pluralismus und dem Zusammentreffen verschiedener Werteorientierungen kommt es nicht von ungefähr, dass der ethische Diskurs Konjunktur hat und wir uns damit auseinandersetzen, wie Menschen trotz aller Unterschiede Gemeinsinn entwickeln, sich engagieren und Verantwortung übernehmen. In einer Konstellation, wie wir sie heute vorfinden, bekommen zwei Fähigkeiten eine Schlüsselfunktion:

- die Fähigkeit, Bindungen kurzfristig aufzubauen und soziale Netzwerke aufrechtzuerhalten und

- die Fähigkeit, Differenzen auszuhalten und Konflikte auszutragen.

Was Gemeinschaft tragfähig macht, ist nicht Einheit und Harmonie, sondern das Gegenteil: Aushandlung von Interessen und konstruktives Konfliktmanagement. Amerikanische Soziologen haben festgestellt, »dass Menschen durch verbale Konflikte eher zusammengehalten werden als durch verbale Übereinstimmung «. Denn: »Im Konfliktfall sind sie zu gründlicherer Kommunikation gezwungen, um die Differenzen auszutragen. «(Coser, zitiert nach Sennett, 1998)

\section{Service Learning als Handlungsanleitung für Sozialkapital}

Es gilt also, die beiden Schlüsselkompetenzen - Bindungen aufzubauen und Interessengegensätze auszugleichen - so früh wie möglich zu entwickeln. Dies geschieht heutzutage nicht mehr automatisch innerhalb verlässlicher Familiensysteme oder über die weiteren Sozialisationsinstanzen Kindergarten, Schule und Freizeit, sondern muss systematisch organisiert werden. Es geht gewissermaßen darum, Gelegenheiten zu schaffen, in denen soziales Lernen geschehen kann (Rauschenbach, 1997).

Vor diesem Hintergrund versteht sich das Konzept Service Learning quasi wie von selbst als Handlungsanleitung für die Bildung von Sozialkapital. Service Lear- ning ist prädestiniert, den Horizont von Studierenden zu weiten, Gemeinsinn und Verantwortungsbereitschaft $\mathrm{zu}$ fördern. Über die Herausforderung, sich in einem bislang ungekannten Terrain zu bewähren, bekommen die Teilnehmenden wichtige Impulse für ihre eigene Persönlichkeitsentwicklung und entfalten Sozialkompetenz. Doch worüber reden wir eigentlich bei Sozialkompetenz?

Ein Blick in die Literatur zeigt unterschiedliche Kompetenzkonzepte, die von geringen zu umfangreichen Ausdifferenzierungen reichen. Durch die von Daniel Golemans angestoßene Debatte um die »Emotionale Intelligenz «, wird die Kategorisierung keineswegs leichter. Der Begriff »Kompetenz « wird vielerorts unscharf verwendet, eine Typisierung scheint nach gegenwärtigem Forschungsstand aussichtslos. Bezug nehmend auf Geißler/Orthey bezeichnet Reinhold Weiß den Begriff Kompetenz als » begriffliche Stopfgans «, (Weiß, 1999, S. 436.) In Anlehnung an die Vorstellungen von Zimmer/Brake kann ein bildhaftes Modell hilfreich sein (vgl. Grafik).
Personale Kompetenz und Sozialkompetenz sind die beiden Grundfertigkeiten, mit sich selbst und anderen Menschen situationsangemessen umzugehen. Dies umfasst einerseits Aspekte der Identitäts- und Persönlichkeitsentwicklung, zum anderen Kompetenzen im Umgang mit anderen Menschen. Personale Kompetenz bezieht sich etwa auf Empathie, Sensibilität, Gerechtigkeitssinn, Toleranz, Verantwortung, Ausdauer, Belastbarkeit, Flexibilität und Eigeninitiative, während Soziale Kompetenz beispielsweise Kommunikationsfähigkeit, Kooperationsfähigkeit, Verhaltenssicherheit, Teamfähigkeit und Konfliktfähigkeit umfasst.

Service-Learning als handlungs- und erfahrungsorientiertes Lernkonzept mit dem Ziel der Persönlichkeitsbildung knüpft an zentrale lerntheoretische Erkenntnisse aus der Pädagogik und Erwachsenenbildung an:

- Das Neue wird mit dem Alten (Bekannten) verknüpft und verglichen.

- Neue Impulse treffen auf den »Resonanzboden« der Erfahrung.

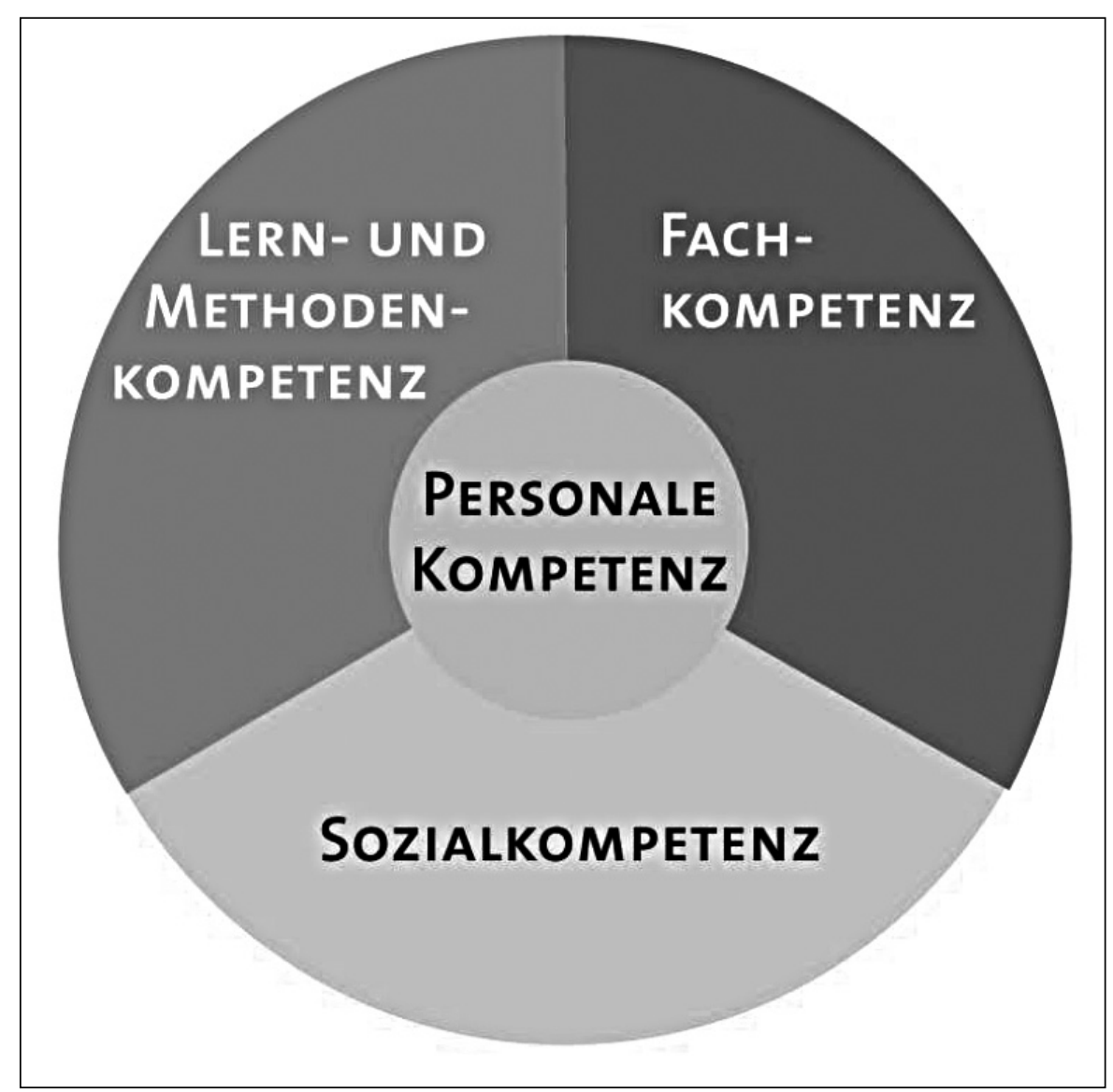

Der Begriff »Kompetenz « wird oft unscharf verwendet; hilfreich ist ein Modell, das verschiedene Aspekte bündelt (in Anlehnung an Zimmer; Brake, 1993, S. 32). 
- Erfahrung speist sich aus unterschiedlichen Quellen, wie die eigene Biografie, Ausbildungen, Familie, Freizeit, Erwerbstätigkeit und außerberufliche, z. B. ehrenamtliche Tätigkeiten (Knoll, 1999, S. 61-79).
Herausforderungen:

- Wie orientiere ich mich rasch in einer für mich fremden Lebenssituation?

- Wie gehe ich mit Menschen um, die für mich ungewohnte Verhaltensmuster zeigen?

\section{»Personale Kompetenz und Sozialkompetenz sind die beiden Grundfertigkeiten, mit sich selbst und anderen Menschen situationsangemessen umzugehen "}

Service Learning kann in unterschiedlichen Formen durchgeführt werden. Wichtig ist, dass die Studierenden beispielsweise aus der Hochschule herausgehen und Erfahrungen in anderen gesellschaftlichen Bereichen machen. Stefan Hansen hat in seiner Dissertation dargestellt, wie »Lernen durch freiwilliges Engagement « in traditionellen Sportvereinen gestaltet werden kann. (Hansen, 2008)

Unsere Erfahrungen speisen sich aus der Organisation von Service-LearningProjekten in sozialen Organisationen und diese eignen sich als Lernfelder für die personale und soziale Kompetenzentwicklung aus drei Gründen besonders gut:

- In sozialen Organisationen findet das Lernen im Gegensatz zu inszenierten »Outdoor-Konstellationen « im realen Leben statt.

- Lernen geschieht im Gegensatz zu Seminar-Settings in sozialen Organisationen ganzheitlich, also mit Herz, Kopf und Hand.

- Lernen in der fremden Lebenswelt »soziale Organisation « hat exemplarischen Charakter. Dies bedeutet, dass soziales Lernen grundsätzlich in jeder Einrichtung möglich ist, die mit Menschen in "besonderen Lebenssituationen « arbeitet.

\section{Herausforderungen und Lernerfahrungen}

Aus unserer langjährigen Praxis lassen sich die Herausforderungen und Lernerfahrungen folgendermaßen generalisieren:
- Verständnis für Menschen in anderen Lebenssituationen entwickeln

- die Arbeitsweise in sozialen Organisationen kennenlernen

- den eigenen Horizont erweitern

- kommunikative Kompetenzen stärken

- die eigene Wahrnehmungsfähigkeit stärken

- das eigene Verhaltensrepertoire erweitern.

(Die Erkenntnis basiert auf der Erfahrung von rund 8.000 Teilnehmenden der unterschiedlichen Projekte unserer Agentur in Schule, Hochschule, betrieblicher Ausbildung und der Führungskräfte-Entwicklung.)

Die Lerneffekte bei Service Learning sind deshalb so nachhaltig, weil die Teilnehmenden mit ihrer eigenen Emotionalität in Kontakt kommen. Betroffenheit wird ausgelöst, wenn sie mit Menschen beispielsweise in schwierigen Lebenssituationen konfrontiert werden. Dies führt $\mathrm{zu}$ einer Relativierung der bis dato empfundenen eigenen Probleme und Schwierigkeiten. Für die Teilnehmenden ist dies vielleicht die wichtigste Erfahrung, die sie beim sozialen Lernen machen. Entscheidend ist dabei das Reflexionsprogramm. Inwieweit die Teilnehmenden tatsächlich neue Erkenntnisse über sich und die anderen gewinnen, steht und fällt damit.

\section{Was versteht man eigentlich unter Service Learning?}

Service-Learning (englisch für: Lernen durch Engagement) ist eine Unterrichtsmethode, die gesellschaftliches Engagement von Schülerinnen und Schülern mit fachlichem Lernen im Unterricht verbinden soll. Service-Learning kombiniert kognitives Lernen (learning) mit der Übernahme von Verantwortung im Schulumfeld (service). Service-Learning wird für alle Altersstufen, Fächer und Schulformen als geeignet betrachtet und auch an Universitäten angewendet.

Die Methode Serivce-Learning (Lernen durch Engagement) kann auch die Lehre an Universitäten bereichern. Beim universitären Service-Learning werden die wissenschaftlichen Inhalte eines Seminars mit gemeinnützigem Engagement der Studierenden verknüpft. Wie für das Schulnetzwerk Lernen durch Engagement heißt auch bei den Hochschulen die Devise: Gemeinsam sind wir stark. Die deutschen Hochschulen, die Service-Learning in ihrer Lehre umsetzen, haben sich am 9. März $2009 \mathrm{zu}-$ sammengeschlossen und das Netzwerk Bildung durch Verantwortung gegründet. Ziel des Netzwerks ist es, Service-Learning an deutschen Hochschulen zu etablieren. Die sechs Gründungsmitglieder sind die Universitäten Duisburg-Essen, Erfurt, Mannheim, Würzburg und des Saarlandes sowie die Fachhochschule Erfurt.

Mit Unterstützung durch die Freudenberg Stiftung ist die Universität Mannheim seit 2003 Vorreiterin bei der Einführung von Service-Learning. Hier fanden 2003 die ersten Service-Learning-Seminare im Fach Pädagogische Psychologie statt, hier verbreitete die daraus entstandene studentische Initiative CampusAktiv die Idee weiter.

Quelle: Internet http://de.wikipedia.org/wiki/Service_Learning 


\section{Service Learning als Beitrag zur Wertevermittlung}

Wir haben gesehen, dass Service Learning ein interessantes, die existierenden Lehrformen ideal ergänzendes außeruniversitäres Lernkonzept darstellt. Wir haben auch über die Notwendigkeit der Entwicklung von Zivilgesellschaften gesprochen, Sozialkapital zu bilden und dafür Sozialisationsinstanzen zur Verfügung zu stellen.
Aus einem weiteren und möglicherweise naheliegenden Grund ist Service Learning für Hochschulen interessant. Studierende sind zukünftige Führungskräfte und Meinungsmacher. Unsere Arbeit mit Führungskräften zeigte uns ein interessantes Phänomen: Die wenigsten Führungskräfte haben gelernt oder sind es gewohnt, systematisch über ihr Verhalten nachzudenken und beschreibend zu reflektieren, was sie warum wie gemacht haben. Also gewissermaßen auf einer Metaebene nachzudenken.

\section{Literatur}

Adloff, Frank: Zivilgesellschaft. Theorie und politische Praxis, Campus Verlag Frankfurt/New York, 2005.

Backhaus-Maul, Holger: Gesellschaftliche Verantwortung von Unternehmen, in: Zeitschrift Aus Politik und Zeitgeschichte APuZ 12/2006, S. 32-38.

Bartsch, Gabriele: Blickwechsel - eine andere Welt erleben, in Karlheinz Geißler/Stephan Laske/Astrid Orthey (Hg.): Handbuch Personalentwicklung, Deutscher Wirtschaftsdienst Wolters Kluwer Verlag, München, 93. Erg.-Lfg., September 2004, 7.26, S. 1-14.

Diess.: Werteorientierte Führung, in: Karlheinz Geißler/Stephan Laske/Astrid Orthey (Hg.): Handbuch PersonalEntwickeln, Deutscher Wirtschaftsdienst Wolters Kluwer Verlag, München, 112. Erg.-Lfg., Mai 2007, 6.60, S. 1--2.

Bourdieu, Pierre: Die feinen Unterschiede. Kritik der gesellschaftlichen Urteilskraft, Suhrkamp Verlag Frankfurt am Main, 2003.

Hansen, Stefan: Lernen durch freiwilliges Engagement. Eine empirische Studie zu Lernprozessen in Vereinen. VS Research Wiesbaden 2008.

Knoll, Jörg: Eigen-Sinn und Selbstorganisation. Zu den Besonderheiten des Lernens von Erwachsenen, in: Kompetenzentwicklung 99. Aspekte einer neuen Lernkultur. Argumente, Erfahrungen, Konsequenzen, Waxmann Verlag Münster/New York/München/Berlin 1999, S.61-79.

Putnam, Robert D. (Hg.): Gesellschaft und Gemeinsinn. Sozialkapital im internationalen Vergleich, Bertelsmann Verlag Gütersloh 2001.

Rauschenbach, Thomas: Zur Notwendigkeit einer neuen Kultur des Sozialen, in: Stark, Werner; Schröer, Andreas; Schubert, Christoph (Hg.): Soziales Lernen in Schule, Betrieb, Jugendarbeit und neuen gesellschaftlichen Organisationsformen, $\mathrm{Pu}$ blikation Evangelische Akademie Bad Boll 1997, S. 30-48.

Sennett, Richard: Der flexible Mensch. Die Kultur des neuen Kapitalismus, Berlin Verlag 1998.

Spoun, Sascha; Wunderlich, Werner: Prolegomena zur akademischen Persönlichkeitsbildung: Die Universität als Wertevermittlerin, in: Sascha Spoun, Werner Wunderlich (Hg.): Studienziel Persönlichkeit. Beiträge zum Bildungsauftrag der Universität heute, Campus Verlag Frankfurt/New York 2005, S. 17-30.

Walzer, Michael: Auf dem Weg zu einer neuen Sozialkultur? In: gleichnamige Broschüre, herausgegeben von den Diakonischen Werken Baden und Württemberg, der Evang. Akademie Bad Boll, der Diözese Rottenburg-Stuttgart und dem Sozialministerium Baden-Württemberg.

Weiß, Reinhold (1999): Erfassung und Bewertung von Kompetenzen - empirische und konzeptionelle Probleme, in: Kompetenzentwicklung 99. Aspekte einer neuen Lernkultur. Argumente, Erfahrungen, Konsequenzen, Waxmann Verlag Münster/New York/München/Berlin 1999, S. 433-493.

Zimmer, Dieter; Brake, Jörg (1993: Ganzheitliche Personalauswahl, Bamberg, S. 32, zitiert nach: KGST-Bericht B 4/1999 (Kommunale Gemeinschaftsstelle Köln).

Diese Fähigkeit, sich neben sich zu stellen und das eigene Verhalten zu reflektieren ist aber unabdingbar, um Handeln und Verhalten überhaupt verändern zu können und dies wiederum ist eine Grundvoraussetzung ethisch orientierten Handelns. Reflexionsfähigkeit wird so zur Kernkompetenz für werteorientierte Führung. Um Reflexionsfähigkeit entwickeln zu können, braucht es spezielle Lernsettings, wie es das Lernkonzept des Service Learning beinhaltet (siehe dazu Bartsch, 2007, S.1-12)

In diesem Sinne können Hochschulen einen wertvollen Beitrag für die Zivilgesellschaft leisten, indem sie die »Grundlagen und Voraussetzungen für individuelle Urteilskraft, Verantwortungsbewusstsein, Führungsbereitschaft und Sozialkompetenz « (Spoun, 2005, S. 23) schaffen. Service Learning ist sicher kein Allheilmittel, aber ein wichtiger Beitrag dafür, dass Studierende an ihrer Hochschule zu Persönlichkeiten heranreifen können.

Der Artikel ist eine gekürzte Fassung des Beitrags der Autorin in dem folgenden Sammelband:

Karsten Altenschmidt, Jörg Miller, Wolfgang Stark (Hg.): Raus aus dem Elfenbeinturm? Entwicklung in Service Learning und bürgerschaftlichem Engagement an deutschen Hochschulen, Beltz Verlag Weinheim und Basel 2009. 